\title{
MECHANOCALORIC EFFECT ON MAGNETOTHERMOELASTIC INTERACTIONS IN A CYLINDRICAL CONDUCTOR CARRYING AN ELECTRIC CURRENT
}

\section{S. Chandrasekharaiah}

To cite this article: D. S. Chandrasekharaiah (1978) MECHANOCALORIC EFFECT ON MAGNETOTHERMOELASTIC INTERACTIONS IN A CYLINDRICAL CONDUCTOR CARRYING AN ELECTRIC CURRENT, Journal of Thermal Stresses, 1:2, 193-198, DOI: $10.1080 / 01495737808926942$

To link to this article: http://dx.doi.org/10.1080/01495737808926942

\section{曲 Published online: 05 Apr 2007.}

Submit your article to this journal $[\pi$

\section{Article views: 12}

Q View related articles $\llbracket$ 


\title{
MECHANOCALORIC EFFECT ON MAGNETOTHERMOELASTIC INTERACTIONS IN A CYLINDRICAL CONDUCTOR CARRYING AN ELECTRIC CURRENT
}

\author{
D. S. Chandrasekharaiah \\ Department of Mathematics \\ Bangalore University \\ Bangalore 560001, India
}

The mechanocaloric effect on magnetothermoelastostatic interactions in a cylindrical conductor carrying a uniform electric current is investigated. It is found that this effect reduces the Joule heating effect and induces nonlinearity into the behavior of stresses. A condition under which the mechanocaloric effect nullifies the Joule effect is also obtained.

\section{INTRODUCTION}

In a recent paper Roetman [1] developed a theory for the basic equations of thermoelasticity, taking into account the mechanocaloric coupling effect. In this theory, unlike the coupled theory [2] and the generalized theory [3], a term involving the body force enters into the equation of heat conduction, and the displacement and thermal fields remain coupled together, even in static deformations. Our purpose in this paper is to utilize the heat equation obtained in [1] to study the interaction between the mechanical field and the thermal field produced in an electrically conducting thermoelastic cylinder permeated by a uniform axial current, assuming that the cylinder undergoes a plane static deformation. This problem was considered earlier by Yuan [4], using the classical form of the heat equation. Our analysis here shows that the inclusion of the mechanocaloric coupling reduces the Joule heating effect and introduces nonlinearity into the behavior of stresses. When the electrical conductivity, the magnetic permeability, the Poisson ratio, and the mechanocaloric coupling constant of the cylinder are connected by a particular relation [Eq. (17) below], the mechanocaloric effect nullifies the Joule effect, and the cylinder temperature does not change. When the mechanocaloric effect is neglected, our results reduce to those obtained in [4].

This work was carried out while the author was visiting the mathematics department of the Cranfield Institute of Technology, Cranfield, England, under the Younger Scientists Exchange Scheme between India and the United Kingdom.

Journal of Thermal Stresses, $1: 193-198,1978$ Copyright $\odot 1978$ by Hemisphere Publishing Corporation $0149-5739 / 78 / 020193-06 \$ 2.75$ 
194 D. S. CHANDRASEKHARAIAH

\section{BASIC EQUATIONS}

Consider a static plane deformation parallel to the $x y$ plane of an electrically conducting thermoelastic solid, due to the presence of an electric current $\mathrm{J}$. The stresses associated with such a deformation are given by [4]

$$
\begin{array}{ll}
\tau_{11}=\frac{\partial^{2} \psi}{\partial y^{2}}+\phi & \tau_{22}=\frac{\partial^{2} \psi}{\partial x^{2}}+\phi \quad \tau_{12}=-\frac{\partial^{2} \psi}{\partial x \partial y} \\
\tau_{33}=\nu\left(\tau_{11}+\tau_{22}\right)-E \alpha T & \tau_{13}=\tau_{23}=0
\end{array}
$$

where $\quad E=$ Young's modulus

$\nu=$ Poisson's ratio

$\alpha=$ coefficient of linear thermal expansion

$T=T(x, y)=$ temperature associated with the deformation

$\phi=\phi(x, y)$ and $\psi=\psi(x, y)$ are governed by the equations

$$
\begin{aligned}
& \nabla^{2} \phi=\mu_{e} J^{2} \\
& \nabla^{4} \psi=\left(\nu^{*}-1\right) \mu_{e} J^{2}-E^{*} \alpha^{*} \nabla^{2} T
\end{aligned}
$$

where $\mu_{e}$ is the magnetic permeability of the body, and

$$
E^{*}=\frac{E}{1-\nu^{2}} \quad \nu^{*}=\frac{\nu}{1-\nu} \quad \alpha^{*}=(1+\nu) \alpha
$$

In the presence of the mechanocaloric effect, the equation governing $T$ in the static case is given by [1]

$$
k \nabla^{2} T+Q+m\left[\operatorname{div} \mathbf{F}+\frac{2 E}{3(1+\nu)} \nabla^{2} e\right]=0
$$

where $\mathcal{e}=$ dilatation

$\mathbf{F}=$ external force per unit volume

$Q=$ strength of internal heat sources

$k=$ thermal conductivity

$m(>0)=$ mechanocaloric coupling constant

In the absence of mechanocaloric coupling $(m=0)$, we readily see from Eq. (4) that the thermal field does not interact with $F$ and $e$, and Eq. (4) reduces to the classical heat equation considered in [4].

The dilatation $e$ is related to $\tau_{11}, \tau_{22}$, and $T$ through the relation (see [4])

$$
e=\frac{1-\nu^{*}}{E^{*}}\left(\tau_{11}+\tau_{22}\right)+2 \alpha^{*} T
$$


This relation yields, with the help of Eqs. (1) and (2),

$$
\nabla^{2} e=\frac{1+\nu^{*}}{E^{*}}\left[\left(1-\nu^{*}\right) \mu_{e} J^{2}+E^{*} \alpha^{*} \nabla^{2} T\right]
$$

If there is no external force other than that due to magnetomechanical interaction, and if there is no heat distribution other than Joule heating, we may take

$$
\mathbf{F}=\mu_{e}(\mathbf{J} \times \mathbf{H}) \quad Q=\frac{1}{\sigma} J^{2}
$$

where $\sigma=$ electrical conductivity of the body

$\mathbf{H}=$ magnetic field associated with $\mathbf{J}$

Using Maxwell's electromagnetic equations and Ohm's law, we may readily verify that

$$
\operatorname{div} \mathbf{F}=\mu_{e} \operatorname{div}(\mathbf{J} \times \mathbf{H})=-\mu_{e} J^{2}
$$

With the aid of Eqs. (5)-(7), Eq. (4) may now be simplified to

$$
\left(k+\frac{2}{3} m E^{*} \alpha^{*}\right) \nabla^{2} T=\left[\frac{1}{3} m\left(1+2 v^{*}\right)-\eta\right] \mu_{e} J^{2}
$$

where $\eta$ is the magnetic viscosity of the body; that is, $\eta=\left(\mu_{e} \sigma\right)^{-1}$.

By eliminating $\nabla^{2} T$ from the second of Eqs. (2) and Eq. (8), we get

$$
\nabla^{4} \psi=\frac{1}{1+2 M}\left[\nu^{*}+\delta-(1+3 M)\right] \mu_{e} J^{2}
$$

where

$$
M=\frac{1}{3} \frac{m E^{*} \alpha^{*}}{k} \quad \delta=\frac{E^{*} \alpha^{*} \eta}{k}
$$

When $\mathbf{J}$ is inown, the first of Eqs. (2) and Eqs. (8) and (9) may be solved under appropriate boundary conditions to determine $\phi, T$, and $\psi$. The stresses then follow from Eqs. (1).

\section{ANALYSIS}

We now turn our attention to the main problem. Let $R$ be the radius of the cylinder, and $J_{0}$ the magnitude of the axial uniform current that the cylinder carries. If we choose the $z$ axis along the axis of the cylinder, we may write $\mathbf{J}=\left(0,0, J_{0}\right)$ in cylindrical coordinates $(r, \theta, z)$ and assume that all field variables depend on $r$ only, because of the axial svmmetry. 
The first of Eqs. (2) and Eq. (8) may now be integrated directly to give

$$
\begin{aligned}
& \phi=\frac{1}{4} \mu_{e} J_{0}^{2} r^{2}+C_{1} \\
& T=\frac{1}{4 E^{*} \alpha^{*}(1+2 M)}\left[\left(1+2 \nu^{*}\right) M-N\right] J_{0}^{2} r^{2}+C_{2}
\end{aligned}
$$

where $C_{1}$ and $C_{2}$ are constants of integration, and

$$
N=\frac{E^{*} \alpha^{*} \eta}{k}=\frac{E^{*} \alpha^{*}}{k \mu_{e} \sigma}
$$

When the mechanocaloric effect is not taken into account, the solution (12) reduces to

$$
T=-\frac{1}{4} \frac{\eta J_{0}^{2} r^{2}}{k}+C_{2}
$$

which agrees with the solution given in [4].

By comparing Eqs. (12) and (14), we may conclude that the mechanocaloric effect makes a significant contribution to the thermal distribution in the cylinder. In the special case of infinite conductivity (i.e., when $\sigma \rightarrow \infty$ ), Eqs. (12) and (14) reduce, respectively, to

$$
\begin{aligned}
& T=\frac{\left(1+2 \nu^{*}\right) M J_{0}^{2} r^{2}}{4 E^{*} \alpha^{*}(1+2 M)}+C_{2} \\
& T=C_{2}
\end{aligned}
$$

We readily see that a thermal distribution of the Fourier type, equivalent to that due to a source of strength

$$
Q=-\frac{\left(1+2 \nu^{*}\right) M}{(1+2 M) E^{*} \alpha^{*}}<0
$$

occurs when the mechanocaloric effect is taken into account, and no thermal distribution occurs otherwise. The mechanocaloric effect thus represents a "heat sink" within the cylinder.

In view of the above analysis, we may conclude that if the cylinder is a good conductor of electricity, the presence of mechanocaloric coupling decreases the Joule heating. In fact, we may verify from Eqs. (10) and (13) that if $\sigma, \nu, \mu_{e}$, and $m$ are connected by the relation

$$
\sigma=\frac{3(1-\nu)}{\mu_{e} m(1+\nu)}
$$


Eq. (12) yields $T=C_{2}$. Therefore, no thermal distribution occurs in the cylinder. This leads us to conclude that when relation (17) holds, the mechanocaloric effect nullifies the Joule effect.

We now take up the solution of Eq. (9). Because of the axial symmetry, this equation reduces to

$$
\frac{1}{r} \frac{d}{d r}\left\{r \frac{d}{d r}\left[\frac{1}{r} \frac{d}{d r}\left(r \frac{d \psi}{d r}\right)\right]\right\}=\frac{\left[\nu^{*}+\delta-(1+3 M)\right] \mu_{e} J_{0}^{2}}{1+2 M}
$$

By integrating this and noting that $\psi$ is to be finite for $r=0$, we get the following solution for $\psi$ :

$$
\psi=a_{1}+a_{2} r^{2}+\frac{\left[\nu^{*}+\delta-(1+3 M)\right] \mu_{e} J_{0}^{2} r^{4}}{64(1+2 M)}
$$

If the boundary of the cylinder is stress free, we have $\tau_{r r}=0$ on $r=R$. Equations (11) and (18), together with this boundary condition, yield the following expressions for stresses:

$$
\begin{aligned}
\tau_{r r} & =\frac{1}{r} \frac{\partial \psi}{\partial r}+\phi=-\frac{\nu^{*}+3+\delta+5 M}{16(1+2 M)}\left(1-\frac{r^{2}}{R^{2}}\right) \mu_{e} J_{0}^{2} R^{2} \\
\tau_{\theta \theta} & =\frac{\partial^{2} \psi}{\partial r^{2}}+\phi=-\frac{\nu^{*}+3+\delta+5 M}{16(1+2 M)}\left[1-\frac{1+3\left(\nu^{*}+\delta\right)-M}{\nu^{*}+\delta+3+5 M} \frac{r^{2}}{R^{2}}\right] \mu_{e} J_{0}^{2} R^{2} \\
\tau_{r \theta} & =0
\end{aligned}
$$

By examining these expressions we see that, unlike the classical situation [4], the stresses developed by the Lorentz force cannot be separated from those caused by the thermal distribution, because of the presence of $1+2 M$ in the common denominators. Hence we may infer that the mechanocaloric coupling introduces nonlinearity into the behavior of stresses in the cylinder. However, this disappears when the mechanocaloric coupling effect tends to vanish, and expressions (19) then reduce to those obtained in [4].

We further verify from Eq. (19) that $\tau_{r r}$ is compressive everywhere and that $\tau_{\theta \theta}$ may be compressive, tensile, or zero as in the classical case. The expression for $\tau_{\theta \theta}$ on the boundary $r=R$ is given by

$$
\left.\tau_{\theta \theta}\right|_{r=R}=-\frac{1}{8}\left(1-\frac{\delta+\nu^{*}-M}{1+2 M}\right) \mu_{e} J_{0}^{2} R^{2}
$$

In the special case when $\sigma, \mu_{e}, \nu$, and $m$ are connected by relation (17), the expressions for $\tau_{r r}$ and $\tau_{\theta \theta}$ given by (19) reduce to

$$
\tau_{r r}=-\frac{\nu^{*}+3}{16}\left(1-\frac{r^{2}}{R^{2}}\right) \mu_{e} J_{0}^{2} R^{2}
$$


198 D. S. CHANDRASEKHARAIAH

$$
\tau_{\theta \theta}=-\frac{\nu^{*}+3}{16}\left(1-\frac{1+3 \nu^{*}}{\nu^{*}+3} \frac{r^{2}}{R^{2}}\right) \mu_{e} J_{0}^{2} R^{2}
$$

It is readily seen that these stresses are due to the action of the Lorentz force only and are independent of the thermal field. This conforms with our earlier observation that when relation (17) holds, the mechanocaloric coupling effect and the Joule effect cancel each other and the cylinder experiences no change in temperature.

\section{REFERENCES}

1. E. L. Roetman, The Mechano-Caloric Effect in Thermoelastic Problems, J. Eng. Math., vol, 10, pp. 355-362, 1976.

2. P. Chadwick, Thermoelasticity, the Dynamic Theory, in I. N. Sneddon \& R. Hill (eds.), Progress in Solid Mechanics, vol. I, North Holland, Amsterdam, pp. 263-328, 1960.

3. H. W. Lord and Y. Shulman, A Generalized Dynamic Theory of Thermoelasticity, J. Mech. Phys. Solids, vol. 15, pp. 299-309, 1967.

4. K. Yuan, Magnetothermoelastic Stresses in an Infinitely Long Cylindrical Conductor Carrying a Uniformly Distributed Axial Current, Appl. Sci. Res., vol. 26, pp. 307-314, 1972.

Communicated by R. S. Dhaliwal on February 3, 1978

Request reprints from D. S. Chandrasekharaiah. 